\title{
La mammographie entre la science, l'irrationnel et la politique
}

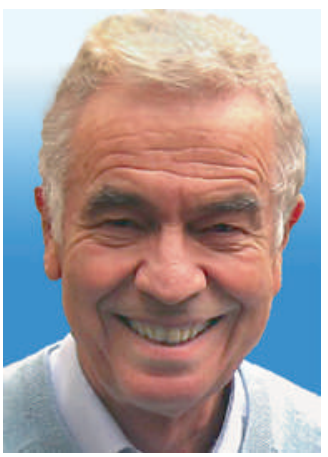

Hans Stalder
Si vous avez toujours été un adepte persuadé de la mammographie ou si vous en êtes un adversaire convaincu, ne continuez pas à lire cet article, car après sa lecture vous ne changerez pas d'opinion. Si vous êtes comme moi, donc indécis, vous pouvez continuer, au risque d'être déçu...

Il y a quatre mois un panel indépendant anglais sous la direction de M. G. Marmot [1] a présenté une méta-analyse concernant le dépistage du cancer mammaire. Tout en soulignant l'incertitude qui règne toujours dans ce domaine, ces experts arrivent aux conclusions suivantes: la réduction du risque de mourir d'un cancer du sein grâce à un dépistage chez des femmes de 50 à 70 ans se situe autour de $20 \%$. Ce chiffre à première vue impressionnant doit être mis en rapport avec le dénominateur: pour 10000 femmes âgées de 50 ans invitées au dépistage pendant 20 ans, 43 décès de cancers du sein seront prévenus; le nombre nécessaire pour sauver une femme est de 235 femmes. Chez les femmes ayant suivi le screening ce nombre est de 180. Cet article discute également la surestimation des diagnostics de néoplasie, des cancers découverts n'aboutissant pas au décès du fait de leur évolution bénigne. Les auteurs arrivent au résultat étonnant que cette probabilité puisse être de $19 \%$, soit 129 femmes sur 10000 faisant le dépistage. Ces femmes subissent une opération et des traitements oncologiques inutiles, le nombre «nécessaire de nuire» étant de 77. Le rapport entre les cancers inutilement traités et des décès prévenus par le dépistage serait donc d'environ 3:1. Il faut aussi se rappeler que pour environ $30 \%$ des mammographies positives la biopsie reste négative, alors qu'elle a causé de l'anxiété et des frais. Un autre article [2], qui compare la prévalence des cancers dans un stade précoce avec celle des cancers avancés, montre que cette dernière a diminué de $8 \%$ seulement du fait du screening (dès l'âge de 40 ans). Selon le calcul des auteurs les opérations et traitements inutiles pourraient alors même s'élever à $31 \%$ !

Dans le même numéro du New England Journal of Medicine trois experts ont été sollicités pour discuter ces résultats [3]: le premier donne des arguments pour le dépistage dès 40 ans, le deuxième dès 50 ans et le troisième recommande d'abandonner le screening. Admettant que ce journal est de haute tenue, vous pouvez donc le citer sans pâlir si vous êtes pour ou si vous êtes contre le screening.
Comment alors décider s'il faut dépister le cancer mammaire quand les avis des scientifiques divergent autant?

Comme souvent quand la science ne donne pas de réponse définitive, les décisions deviennent irrationnelles. On est pour ou contre. Et aux croyances on peut appliquer le théorème de Bayes [4] utilisé habituellement pour préciser un diagnostic: si vous croyez à une chose très fortement - si le diagnostic a priori est quasiment certain -, un nouvel argument - un nouveau test - ne va pas changer votre opinion - ne changera pas le diagnostic a posteriori. Autrement exprimé: c'est seulement si vous hésitez qu'une nouvelle donnée vous convaincra.

En Suisse, ce sont nos gouvernements ou parlements cantonaux qui prennent des décisions sur la mammographie. A défaut d'arguments scientifiques clairs, ils votent sur des arguments émotionnels ou politiciens: ils sont pour afin ne pas être accusés de sexisme, ou contre pour ne pas être soupçonnés d'étatisme. Ainsi il n'est pas étonnant que les prises en charge de la mammographie dépendent de la couleur politique et diffèrent d'un canton à l'autre. Ce n'est pas vraiment une solution idéale ...

Pour sortir de l'irrationnel dans un domaine aussi important que le dépistage généralisé, on doit trouver d'autres approches. Ce ne sont pas seulement les données épidémiologiques qui comptent. Il y a par exemple aussi la question éthique à considérer: est-il acceptable d'opérer et de traiter des femmes inutilement - primum nil nocere! - même si on en sauve d'autres?

Ce genre de réflexion ne peut se faire que dans un comité «de sages», insensibles aux arguments émotionnels et politiques, où sont représentés les aspects médical, épidémiologique, éthique, juridique et économique. Une institution comme le Swiss Medical Board me semble répondre à cette exigence. Les spécialistes devraient y être consultés, mais ne pas participer à la décision finale car - avouons-le - comme spécialiste, chacun de nous a ses propres petits intérêts à défendre. Espérons que le Medical Board ne soit pas découragé par les réactions tumultueuses à leur rapport sur le dépistage du cancer prostatique!

Hans Stalder* 